\title{
ON THE ALMOST EVERYWHERE EXISTENCE OF THE ERGODIC HILBERT TRANSFORM
}

\author{
DIEGO GALLARDO AND F. J. MARTÍN-REYES
}

(Communicated by R. Daniel Mauldin)

\begin{abstract}
Let $(X, \mathfrak{M}, \mu)$ be a finite measure space, $T$ an invertible measurepreserving transformation and $v$ a positive measurable function. For $p=1$, we prove that the ergodic Hilbert transform $H f(x)=\lim _{n \rightarrow \infty} \sum_{i=-n}^{n}{ }^{\prime} f\left(T^{i} x\right)$ $/ i$ exists a.e. for every $f$ in $L^{1}(v d \mu)$ if and only if $\inf _{i \geq 0} v\left(T^{i} x\right)>0$ a.e. We also solve the problem for $1<p \leq 2$. In this case the condition is $\sup _{k \geq 1} k^{-1} \sum_{i=0}^{k-1} v^{-1 /(p-1)}\left(T^{i} x\right)<\infty$ a.e. If the transformation $T$ is ergodic, the characterizing conditions become that $1 / v \in L^{\infty}$ and $v^{-1 /(p-1)} \in$ $L^{1}(\mu)$, respectively. These characterizations, together with some recent results, give, for $1 \leq p \leq 2$, that $H f(x)$ exists a.e. for every $f$ in $L^{p}(v d \mu)$ if and only if the sequence of the Césàro-averages $k^{-1}\left(f(x)+f(T x)+\ldots f\left(T^{k-1} x\right)\right)$ converge a.e. for every $f$ in $L^{p}(v d \mu)$. This equivalence has recently been obtained by Jajte for a unitary operator, not necessarily positive, acting on $L^{2}$.
\end{abstract}

\section{INTRODUCTION}

Let $(X, \mathfrak{M}, \mu)$ be a $\sigma$-finite measure space and let $T$ be an invertible measure preserving transformation from $X$ into itself. We consider the ergodic Hilbert transform, associated to $T$, defined by

$$
H f(x)=\lim _{n \rightarrow \infty} \sum_{i=-n}^{n} \frac{f\left(T^{i} x\right)}{i},
$$

where $f$ denotes a measurable function and the prime denotes omission of the 0 th term.

In [1], Cotlar shows that if $\mu(X)=1$, then, the ergodic Hilbert transform of each function $f \in L^{1}(\mu)$ exists almost everywhere. The above also remains true when $\mu(X)=\infty$. (For a simple proof see [8]).

Our aim in this paper is to characterize those positive measurable functions $v$ for which there exists $H f$, in the pointwise sense, for every $f \in L^{p}(v d \mu), 1 \leq$ $p<\infty$.

Received by the editors January 15, 1988 and, in revised form May 9, 1988.

1980 Mathematics Subject Classification (1985 Revision). Primary 28D05; Secondary 47A35.

Key words and phrases. Almost everywhere convergence, Cesàro-averages, ergodic Hilbert transform, ergodic maximal Hilbert transform, ergodic maximal operator, measure preserving transformations, weights.

This research has been supported by a C.A.Y.C.I.T. Grant (PB85-0434). 
In the case $p=1$ and $\mu(X)<\infty$, we prove that the characterizing condition is given by $\inf _{i \geq 0} v\left(T^{i} x\right)>0$ a.e., which is equivalent, in this case, to $\inf _{-\infty<i<\infty} v\left(T^{i} x\right)>0$ a.e.. However, the last condition is only sufficient but not necessary for the existence of the ergodic Hilbert transform when $\mu(X)=\infty$.

In the case $p>1$ and $\mu(X)<\infty$, we consider the ergodic maximal operator

$$
M f(x)=\sup _{n \geq 1}\left|A_{n} f\right|
$$

associated to the Césàro-averages

$$
A_{n} f(x)=\frac{1}{n} \sum_{i=0}^{n-1} f\left(T^{i} x\right)
$$

and we show that condition $M v^{-1 /(p-1)}(x)<\infty$ a.e. implies the existence of $H f(x)$ a.e. for each $f \in L^{p}(v d \mu)$ and moreover such a condition is also necessary when $1<p \leq 2$.

In what follows, we will alway assume that $T: X \rightarrow X$ is an invertible measure preserving transformation, $v$ is a positive measurable function and $H, M, A_{n}$ are the operators defined by (1.1)-(1.3), respectively. On the other hand, throughout this paper, we denote by $H^{*}$ the ergodic maximal Hilbert transform

$$
H^{*} f(x)=\sup _{n \geq 1}\left|\sum_{i=-n}^{n} \frac{f\left(T^{i} x\right)}{i}\right|
$$

and by $f^{*}$ the ergodic maximal function

$$
f^{*}=\sup _{n, m \geq 0}\left|\frac{1}{n+m+1} \sum_{i=-n}^{m} f\left(T^{i} x\right)\right| .
$$

Finally, we will consider two sets as equals if they agree up to a set of measure zero.

\section{THE MAIN RESULT}

Theorem 2.1. Let $(X, \mathfrak{M}, \mu)$ be a finite measure space and $1 \leq p \leq 2$. The following statements are equivalent:

(a) There exists $H f(x)$ a.e. for every $f \in L^{p}(v d \mu)$.

(b) $H^{*} f(x)<\infty$ a.e. for every $f \in L^{p}(v d \mu)$.

(c) There exists a positive measurable function $u$ such that for every $f$ and $\lambda>0$

$$
\int_{\left\{x \in X: H^{*} f(x)>\lambda\right\}} u d \mu \leq \lambda^{-p} \int_{X}|f|^{p} v d \mu .
$$

(d) $\inf _{-\infty<i<\infty} v\left(T^{i} x\right)>0$ a.e. if $p=1 ;\left(v^{-1 /(p-1)}\right)^{*}(x)<\infty$ a.e. if $p>1$. 
(e) $\inf _{i \geq 0} v\left(T^{i} x\right)>0$ a.e. if $p=1 ; M v^{-1 /(p-1)}(x)<\infty$. a.e. if $p>1$.

Proof. It is clear that (a) implies (b) and (d) implies (e). On the other hand, (b) implies (c) by Nikishin's theorem (see [2]). Then, it remains to prove (e) $\Rightarrow(a)$ and $(\mathrm{c}) \Rightarrow(\mathrm{d})$.

Proof of (e) $\Rightarrow(\mathrm{a})$. If $p=1$, it follows from (e) that $X=\bigcup_{s \geq 1} X_{s}$, where

$$
X_{s}=\left\{x \in X: \sup _{i \geq 0} v^{-1}\left(T^{i} x\right)<s\right\} .
$$

The $X_{s}$ are absorbing sets, that is $X_{s} \subset T^{-1}\left(X_{s}\right)$, and, since $\mu(X)<\infty$, we have that the $X_{s}$ are invariant sets, i.e., $T\left(X_{s}\right)=X_{s}$. On the other hand, it is easy to see that $L^{1}\left(X_{s}, v d \mu\right) \subset L^{1}\left(X_{s}, \mu\right)$ and thus (a) follows from this and Cotlar's result.

Now, let $p>1$ and $\sigma=v^{-1 /(p-1)}$. Since $\limsup _{n \rightarrow \infty} A_{n} \sigma(x)<\infty$ a.e., we decompose $X$ into the invariant sets $X_{s}=\left\{x \in X: \limsup _{n \rightarrow \infty} A_{n} \sigma(x)<s\right\}$.

For each positive integer $N$ let $\sigma_{N}=\min (\sigma, N)$. Since $\sigma_{N} \in L^{1}\left(X_{s}, \mu\right)$ and $\mu\left(X_{s}\right)$ is finite, it follows from Birkhoff's ergodic theorem that there exists an invariant function $\sigma_{N}^{0}$ in $L^{1}\left(X_{s}, \mu\right)$ such that $\lim _{k \rightarrow \infty} A_{k} \sigma_{N}(x)=\sigma_{N}^{0}(x)$ for almost every $x$ in $X_{s}$. Moreover

$$
\int_{X_{s}} \sigma_{N} d \mu=\int_{X_{s}} \sigma_{N}^{0} d \mu
$$

since $X_{s}$ is invariant. Therefore,

$$
\int_{X_{s}} \sigma_{N} d \mu \leq \int_{X_{s}} \lim \sup _{k \rightarrow \infty} A_{k} \sigma d \mu<s \mu\left(X_{s}\right) .
$$

Then, it follows from the Lebesgue's monotone convergence theorem that $\sigma \in$ $L^{1}\left(X_{s}, \mu\right)$.

On the other hand, Hölder's inequality shows

$$
\int_{X_{s}}|f| d \mu \leq\left(\int_{X_{s}}|f|^{p} v d \mu\right)^{1 / p}\left(\int_{X_{s}} \sigma d \mu\right)^{1 / p^{\prime}},
$$

where $p^{\prime}$ is the conjugate exponent of $p$. Therefore, $L^{p}\left(X_{s}, v d \mu\right) \subset L^{1}\left(X_{s}, \mu\right)$ and, thus, we obtain (a).

Proof of $(\mathrm{c}) \Rightarrow(\mathrm{d})$. In order to prove this implication we will need the following definition and the following Lemma proved in [4].

Definition 2.2. Let $s$ be a positive integer. The measurable set $B \subset X$ is the base (with respect to $T$ ) of an ergodic rectangle of length $s$ if $T^{i} B \cap T^{j} B=$ $\varnothing, i \neq j, 0 \leq i, j \leq s-1$. In such case, the set $\mathfrak{R}=\bigcup_{0 \leq i \leq s-1} T^{i} B$ will be called ergodic rectangle with base $B$ and length $s$.

Lemma 2.3. Let $Y$ be a measurable subset of $X$ and let $k$ be a positive integer. Then, there exists a countable family $\left\{B_{i}, i \in \mathbb{Z}^{+}\right\}$of sets of finite measure such that: 
(i) $Y=\bigcup_{i=0}^{\infty} B_{i}$.

(ii) $B_{i} \cap B_{j}=\varnothing$ if $i \neq j$.

(iii) For every $i, B_{i}$ is the base of an ergodic rectangle of length $s(i) \leq k$ and such that if $s(i)<k$, then $T^{s(i)} A=A$ for every measurable set $A \subset B_{i}$.

For every positive integer $k$ let $\left\{B_{i, k}, i \in \mathbb{Z}\right\}$ be the sequence given by Lemma 2.3 for an integer sufficiently large, for example $4 k$, and the set $X$.

We consider $X=Y \cup Z$, where

$$
Y=\bigcap_{k=1}^{\infty}\left(\bigcup_{\{i: s(i, k)=4 k\}} B_{i, k}\right)
$$

and

$$
Z=\bigcup_{k=1}^{\infty}\left(\bigcup_{\{i: s(i, k)<4 k\}} B_{i, k}\right)
$$

First, we notice that $\inf _{-\infty<i<\infty} v\left(T^{i} x\right)>0$ for almost every $x$ in $Z$ and, therefore, $\left(v^{-1 /(p-1)}\right)^{*}(x)<\infty$ a.e. $x$ in $Z$ if $p>1$, even if (c) does not hold.

Let $B_{i, k}$ be a base of a rectangle with length $s(i, k)<4 k$ for some integer $k$. We have

$$
B_{i, k}=\bigcup_{\left(n_{1}, n_{2}, \ldots, n_{s(i, k)} \in \mathbf{Z}^{s(i, k)}\right.} D_{n_{1}, n_{2}, \ldots, n_{s(i, k)}},
$$

where

$$
\begin{array}{r}
D_{n_{1}, n_{2}, \ldots, n_{s(i, k)}}=\left\{x \in B_{i, k}: 2^{n_{1}} \leq v(x)<2^{n_{1}+1}, 2^{n_{2}} \leq v(T x)<2^{n_{2}+1}, \ldots,\right. \\
\left.2^{n_{s(i, k)}} \leq v\left(T^{s(i, k)-1} x\right)<2^{n_{s(i, k)+1}}\right\}
\end{array}
$$

By Lemma (2.3) we have that $T^{s(i, k)} A=A$ where $A=D_{n_{1}, n_{2}, \ldots, n_{s(i, k)}}$ and, therefore for almost every $x$ in $A$

$$
\inf _{-\infty<i<\infty} v\left(T^{i} x\right) \geq \min \left\{2^{n_{j}}, 1 \leq j \leq n_{s(i, k)}\right\}>0 .
$$

Thus, by (2.5) and (2.6) we get $\inf _{-\infty<i<\infty} v\left(T^{i} x\right)>0$ a.e. in $Z$.

Now, we will prove that condition (d) holds for almost every $x$ in $Y$. We may assume without loss of generality that $u \in L^{1}(\mu)$ and $u \leq v$.

Let us fix a positive integer $k$ and let $B_{i, k}$ be a base of a rectangle with length $s(i, k)=4 k$. If $p=1$ we consider any subset $E$ of $B_{i, k}$ with positive measure and any integer $h$. It is easy to see that if $x \in E$ and $j$ is an integer such that $h+1 \leq j \leq h+k$, then $H^{*} \chi_{T^{h} E}\left(T^{j} x\right) \geq 1 / k$, where $\chi_{T^{h} E}$ denotes the characteristic function of $T^{h} E$. Therefore, it follows from (c) that

$$
\int_{\bigcup_{j=h+1}^{h+k} T^{j} E} u d \mu \leq k \int_{T^{h} E} v d \mu
$$


for every integer $h$. Since $T$ preserves the measure, it follows from (2.7) that

$$
\int_{E} A_{k+1} u\left(T^{h} x\right) d \mu(x) \leq \int_{E} v\left(T^{h} x\right) d \mu(x)
$$

and, therefore, for a.e. $x \in B_{i, k}$ we have

$$
A_{k+1} u\left(T^{h} x\right) \leq v\left(T^{h} x\right), \text { for every integer } h .
$$

Now, it is easy to see that (2.8) holds for almost every $x$ in $Y$ and any $k$.

On the other hand, Birkhoff's ergodic theorem asserts that the averages $A_{k+1} u$ converge a.e. to a positive invariant function $u_{0}$. Thus, if we let $k$ tend to infinity in (2.8) we get $0<u_{0}(x) \leq v\left(T^{h} x\right)$, for a.e. $x$ in $Y$ and any $h$, which proves (d), in the case $p=1$.

Now, we consider $p>1$ and $\sigma=v^{-1 /(p-1)}$. Then, for each integer $r$ we define

$$
E_{i, k, r}=\left\{x \in B_{i, k}: 2^{r+1}<A_{k} \sigma(x) \leq 2^{r+2}\right\}
$$

and for a measurable set $E \subset E_{i, k, r}$, with $\mu(E)>0$, let $\mathfrak{R}$ be the rectangle with base $E$ and length $k$, i.e., $\mathfrak{R}=\bigcup_{0 \leq i \leq k-1} T^{i} E$.

If $f$ is a non-negative function with support in $\mathfrak{R}$ we have $H^{*} f\left(T^{j} x\right) \geq$ $2^{-1} A_{k} f(x)$ for $x \in E$ and $k \leq j \leq 2 k-1$. Then, for $f=\sigma \chi_{\mathfrak{R}}$ we obtain

$$
Q=\bigcup_{j=k}^{2 k-1} T^{j} E \subset\left\{x \in X: H^{*}\left(\sigma \chi_{\mathfrak{R}}\right)(x)>2^{r}\right\}
$$

and, consequently, it follows from (c) that $2^{r p} \int_{Q} u d \mu \leq \int_{\mathfrak{R}} \sigma d \mu$, that is,

$$
2^{r p} \int_{E} \sum_{i=k}^{2 k-1} u\left(T^{i} x\right) d \mu(x) \leq \int_{E} \sum_{i=0}^{k-1} \sigma\left(T^{i} x\right) d \mu(x) .
$$

Therefore, by the definition of $E_{i, k, r}$ we have

$$
\int_{E}\left(A_{k} \sigma(x)\right)^{p} A_{k} u\left(T^{k} x\right) d \mu(x) \leq 4^{p} \int_{E} A_{k} \sigma(x) d \mu(x),
$$

and, thus, we obtain that there exists a constant $C$ such that for almost every $x$ in $B_{i, k}$

$$
\left(A_{k} \sigma(x)\right)^{p-1} A_{k} u\left(T^{k} x\right) \leq C,
$$

and, therefore, for a.e. $x$ in $Y$ and every positive integer $k$.

On the other hand, since $u \in L^{1}(\mu)$ and $\mu(X)<\infty$, we get

$$
\lim _{k \rightarrow \infty} A_{k} u\left(T^{k} x\right)>0
$$

a.e. and, therefore, for almost every in $Y$ there exists a real $\alpha(x)>0$ such that for every positive integer $k$

$$
\left(A_{k} \sigma(x)\right)^{p-1} \leq C / \alpha(x)
$$


It is obvious that we can obtain an inequality as (2.9) with $T^{-1}$ instead of $T$. Therefore, we have $\sigma^{*}(x)<\infty$ a.e. in $Y$ and, thus, the proof of Theorem 2.1 is complete.

Remarks. 1. In the case $\mu(X)=\infty$, condition (d), for $p=1$, of Theorem 2.1 implies the existence of the ergodic Hilbert transform, since the sets $\{x \in$ $\left.X: \sup _{-\infty \leq i \leq \infty} v^{-1}\left(T^{i} x\right)<s\right\}$ are invariant and, then, it is enough to take these sets instead of $X_{s}$ in the proof of (e) $\Rightarrow(\mathrm{a})$ in Theorem 2.1. However, (a) does not imply (d), as the following example shows.

(2.10) Example. Let $X$ be the set of the integers with the counting measure $\mu, T$ the shift transformation $T k=k+1$ and $v$ the function defined by $v\left(2^{n}\right)=v\left(-2^{n}\right)=1 / n$ for every positive integer $n$ and $v(x)=1$ otherwise.

Let $f$ be a nonnegative function in $L^{1}(v d \mu)$. We define the function $g$ given by $g(x)=f(x)$ if $x= \pm 2^{n}$ for some $n$ and $g(x)=0$ otherwise. Since $f-g$ is in $L^{1}(\mu)$ there exists $H(f-g)(x)$ for every $x$. We will now prove that $H g(x)$ also exists for every $x$.

Let $x \geq 2$ and $s$ an integer such that $2^{s-1} \leq x<2^{s}$. For each positive integer $k$ let

$$
a_{k}(x)=\sum_{i=1}^{k} \frac{g(x+i)}{i} \text { and } b_{k}(x)=\sum_{i=1}^{k} \frac{g(x-i)}{i} .
$$

We have that $a_{k}(x)=\sum_{j=s}^{n} g\left(2^{j}\right) /\left(2^{j}-x\right)$, for some integer $n$, and therefore for every $k$

$$
a_{k}(x)=\sum_{j=2}^{n} g\left(2^{j}\right) v\left(2^{j}\right) \frac{j}{2^{j}-x} \leq\|f\|_{1, v d \mu} \sum_{j=s}^{\infty} \frac{j}{2^{j}-x}<\infty,
$$

which proves that the sequence $\left\{a_{k}(x)\right\}$ converges as $k \rightarrow \infty$.

On the other hand, for $k$ sufficiently large, $b_{k}(x)$ is dominated by a sum of the type

$$
\sum_{j=1}^{s-1} g\left(2^{j}\right) /\left(x-2^{j}\right)+\sum_{j=1}^{n} g\left(-2^{j}\right) /\left(x+2^{j}\right),
$$

for some positive integer $n$, and since

$$
\sum_{j=1}^{n} g\left(-2^{j}\right) /\left(x+2^{j}\right) \leq\|f\|_{1, v d \mu} \sum_{j=1}^{\infty} j /\left(x+2^{j}\right)<\infty,
$$

we obtain that $\left\{b_{k}(x)\right\}$ also converges as $k \rightarrow \infty$.

Therefore, we get that there exists $H g(x)$ for $x \geq 2$. The existence for $x<2$ can be proved in a similar way. Thus, we conclude that $H f(x)$ exists for every $x$ and any $f \in L^{1}(v d \mu)$ but inf ${ }_{-\infty<i<\infty} v\left(T^{i} x\right)=0$. Furthermore, in this example, we have that $\inf _{i \leq 0} v\left(T^{i} x\right)=0$ and $\inf _{i \geq 0} v\left(T^{i} x\right)=0$, which shows that condition " $\inf _{i \leq 0} v\left(T^{i} x\right)>0$ or $\inf _{i \geq 0} v\left(T^{i} x\right)>0$ " is not necessary for the existence of the ergodic Hilbert transform in the case $\mu(X)=\infty$. 
2. If the transformation $T$ is ergodic, condition (d) and (e) of Theorem 2.1 , in the case $p=1$, become that $1 / v \in L^{\infty}$, since the function $u(x)=$ inf $_{-\infty<i<\infty} v\left(T^{i} x\right)$ is invariant and, therefore, essentially constant. In this case, the proof of $(\mathrm{e}) \Rightarrow(\mathrm{a})$ is trivial since the inclusion $L^{p}(v d \mu) \subset L^{1}(\mu)$ is obvious. If $T$ is ergodic and $p>1$, conditions (d) and (e) reduce to saying that $\sigma=v^{-1 /(p-1)} \in L^{1}(\mu)$, since if (e) holds, then $X$ agrees with some invariant set $X_{s}=\left\{x \in X: \limsup _{n \rightarrow \infty} A_{n} \sigma(x)<s\right\}$ and, on the other hand, $\sigma \in L^{1}\left(X_{s}, \mu\right)$, as we saw in the proof of $(\mathrm{e}) \Rightarrow(\mathrm{a})$. Moreover, if $T$ is ergodic and the measure space is nonatomic, then the set $Z$ defined in (2.5) is empty, since it follows easily from Lemma 2.3 that, for every positive integer $k$, the set $X$ can be written as a countable union of bases of rectangles of length $4 k$ (see Corollary 2.12 in [4]).

3. In the case $p>1$, the proofs of $(e) \Rightarrow(a)$ and (c) $\Rightarrow(d)$ do not use that $p \leq 2$. The condition $p \leq 2$ is only used in implication $(b) \Rightarrow(c)$, when we apply Nikishin's theorem. Thus, we leave as an open problem to see whether or not condition (a) implies conditions (d) when $p>2$. On the other hand, the result (e) $\Rightarrow$ (a) can be extended to the context of Orlicz spaces.

We consider an $N$-function $\phi$, that is, a function $\phi(s)=\int_{0}^{s} \varphi$ where $\varphi:[0, \infty) \rightarrow \mathbf{R}$ is continuous from the right, nondecreasing such that $\varphi(s)>$ $0, s>0, \varphi(0)=0$ and $\varphi(s) \rightarrow \infty$ for $s \rightarrow \infty$, and, associated to $\phi$, we consider the Orlicz class $L_{\phi}(\mu)=\left\{f \in \mathfrak{M}: \int_{X} \phi(|f|) d \mu<\infty\right\}$, where $\mathfrak{M}$ denotes the space of measurable and a.e. finite functions from $X$ to $\mathbb{R}$ (or to $\mathbb{C}$ ). Thus, if $\phi(s)=s^{p}, p>1$, then $L_{\phi}(\mu)=L^{p}(\mu)$.

Associated to $\varphi$ we have $\rho:[0, \infty) \rightarrow \mathbf{R}$ defined by $\rho(t)=\sup \{s: \varphi(s) \leq t\}$ (the generalized inverse function of $\varphi$ ), which has the same aforementioned properties of $\varphi$. Then, the $N$-function $\psi$ defined by $\psi(t)=\int_{0}^{t} \rho$ is called the complementary $N$-function of $\phi$. Thus, if $\phi(s)=p^{-1} s^{p}, p>1$, then $\psi(t)=q^{-1} t^{p^{\prime}}$ where $p^{\prime}$ is the conjugate exponent of $p$.

Young's inequality asserts that $s t \leq \phi(s)+\psi(t)$ for $s, t \geq 0$, equality holding if $t=\varphi(s)$ or else $s=\rho(t)$. (See II-13 in [7].)

Then, the proof of (e) $\Rightarrow(\mathrm{a})$, in the case $p>1$, can be easily adapted, taking $\sigma=\rho(1 / v)$ instead of $\sigma=v^{-1 /(p-1)}$, in the following way:

We consider $X_{s}=\left\{x \in X: \lim \sup _{n \rightarrow \infty} A_{n} \sigma(x)<s\right\}$ and we obtain that $\sigma \in L^{1}\left(X_{s}, \mu\right)$ using the same arguments used there. On the other hand, taking into account Young's inequality and the equality cases in this inequality, we have

$$
\int_{X_{s}}|f| d \mu \leq \int_{X_{s}} \phi(|f|) v d \mu+\int_{X_{s}} \psi(1 / v) v d \mu \leq \int_{X_{s}} \phi(|f|) v d \mu+\int_{X_{s}} \sigma d \mu
$$

and therefore we obtain $L_{\phi}\left(X_{s}, v d \mu\right) \subset L^{1}\left(X_{s}, \mu\right)$. Thus, we conclude that if $(X, \mathfrak{M}, \mu)$ is a finite measure space and $\phi$ an $N$-function, the condition $M \rho(1 / v)(x)<\infty$ a.e. implies that $H f(x)$ exists a.e. for every $f \in L_{\phi}(v d \mu)$. 
We remark that, if $T$ is ergodic, the condition $M \rho(1 / v)(x)<\infty$ a.e. becomes that $\rho(1 / v) \in L^{1}(\mu)$.

4. The characterizations of the positive functions $v$ for which the Cesàroaverages $A_{n} f$ converge a.e. for every $f$ in $L^{p}(v d \mu)$ are studied in [5] for $p=1$ and in [6] for $1<p<\infty$, with $T$ not necessarily invertible and assuming $\mu(X)<\infty$. The characterizing conditions are precisely the conditions included in (e) of Theorem 2.1. Therefore, we have the following corollary:

Corollary 2.11. Let $(X, \mathfrak{M}, \mu)$ be a finite measure space and $1 \leq p \leq 2$. Then, $H f(x)$ exists a.e. for every $f \in L^{p}(v d \mu)$ if, and only if, the Cesàro-averages $A_{n} f$ converge a.e. for every $f \in L^{p}(v d \mu)$.

The above result was proved in [3] for unitary operators (not necessarily positive) acting on $L^{2}$. Corollary 2.12 is also closely related to the following result, obtained by Wos in [9].

Theorem. Let $(X, \mathfrak{M}, \mu)$ be a finite measure space and $T$ an ergodic invertible measure preserving transformation from $X$ into itself. If $f$ is a measurable function such that the averages $A_{n} f$ converge a.e., then, the ergodic Hilbert transform $H f(x)$ exists a.e.

Our result is similar to Wos' but as far as we know the converse of this theorem is an open problem. Of course, there are at least two more differences: the transformation in the paper of Wos is ergodic but he assumes the existence of the limit for a single function and not for all the functions in a certain class $L^{p}(v d \mu)$ as we do.

\section{REFERENCES}

1. M. Cotlar, A unified theory of Hilbert transforms and ergodic theorems, Rev. Math. Cuyana 1 (1955), 105-167.

2. J. E. Gilbert, Nikishin-Stein theory and factorization with applications, Proc. Sympos. Pure Math. 35 (1979), 233-267.

3. R. Jajte, On the existence of the ergodic Hilbert transforms, Ann. Probab. 15 (1987), 831-835.

4. F. J. Martin-Reyes, Inequalities for the ergodic maximal function and convergence of the averages in weighted $L^{p}$-spaces, Trans. Amer. Math. Soc. 296 (1986), 61-82.

5. F. J. Martin-Reyes and A. de la Torre, Weighted weak type inequalities for the ergodic maximal function and the pointwise ergodic theorem, Studia Math. 87 (1987), 33-46.

6. _ On the almost everywhere convergence of the ergodic averages, to appear in Ergodic Theorey and Dynamical Systems.

7. J. Musielak, Orlicz spaces and modular spaces, Springer-Verlag, Berlin, 1983.

8. K. Petersen, Another proof of the existence of the ergodic Hilbert transform, Proc. Amer. Math. Soc. (1983), 39-43.

9. J. Wos, A remark on the existence of the ergodic Hilbert Transform, Colloq. Math. (1987).

Análisis Matemático, Facultad de Ciencias, 29071-Málaga, Universidad de Málaga, SPAIN 